\title{
Effect of Amino Acids on Coremium Development in Penicillium claviforme
}

\author{
By S. C. WATKINSON \\ Department of Botany, University of Oxford, Oxford $O X_{\mathrm{I}}{ }_{3} R A$
}

(Received 8 April 1977)

\begin{abstract}
Amino acids added to the growth medium stimulated development of coremium primordia in mycelium of Penicillium claviforme. Casein hydrolysate, L-asparagine, L-serine, L-glutamine, L-proline, L-hydroxyproline, L-glutamate and glycine accelerated development and also increased the final number of primordia per unit area of mycelium. A nearly linear relationship existed between the logarithm of casein hydrolysate concentration and the numbers of primordia developed, and a similar relationship was also shown with glutamate as nitrogen source. Mycelium developing from spores sown on agar plates showed some sites for coremium development within a few hours of germination, although most sites were not established until about $24 \mathrm{~h}$ later when the germlings had fused to form a network. At least $27 \mathrm{~h}$ exposure was required for amino acids to stimulate primordium development in $24 \mathrm{~h}$-old mycelium. Cycloheximide, glucosamine and nystatin promoted primordium development at concentrations inhibiting hyphal growth; 2-deoxyglucose inhibited primordium formation. It was concluded that possible sites for coremia develop into primordia as a result of changes in nitrogen metabolism, and that local variations in the concentrations of certain amino acids or their metabolites may decide the pattern in which primordia form in the colony.
\end{abstract}

\section{INTRODUCTION}

The development of coremia by Penicillium claviforme Bainier is convenient for the study of differentiation in fungi because the structures formed are simple compared with perfect stages, and their sites of origin and earliest stages can be located and observed.

Primordium formation involves the development of photosensitivity and of the capacity for hyphal aggregation; it also involves the accumulation of nutrient reserves, since primordia become capable of independent, endotrophic development into sporulating coremia (Carlile et al., 196I; Watkinson, 1975).

There is evidence that nutrient concentration regulates initiation of primordia. When undifferentiated mycelium is transferred to nutrient medium at a series of concentrations, the number of primordia produced is proportional to the logarithm of nutrient concentration (Watkinson, 1975). The characteristic concentric circles of coremia produced when a colony grows from a point inoculum can be accentuated and regulated by various factors (Faraj Salman, 1970, I97I $a, b$ ), but these may be overridden by high concentrations of nutrients. In this work I attempted to identify the components of the medium that stimulate development and to trace the time course of primordium initiation in the hope of discovering what developmental processes are affected. 


\section{METHODS}

The culture method used to observe nutrient effects on primordium formation produced a synchronouslydeveloping mycelial mat. Identical samples could easily be transferred to different media and observed microscopically in situ. The standard medium contained (g per litre tap water): sucrose, 12.5; Oxoid acid casein hydrolysate, 3.2; $\mathrm{KH}_{2} \mathrm{PO}_{4}, \mathrm{I} ; \mathrm{MgSO}_{4} \cdot 7 \mathrm{H}_{2} \mathrm{O}$, 0.5; $\mathrm{FeSO}_{4} \cdot 7 \mathrm{H}_{2} \mathrm{O}$, 0.0I; and Oxoid no. 3 agar, 20; the $\mathrm{pH}$ after autoclaving was $6 \cdot 5$. Portions $(20 \mathrm{ml})$ of this medium were poured into $9 \mathrm{~cm}$ plastic Petri dishes. Cellophane circles were boiled in distilled water, autoclaved and placed on the surface of the medium. Spore suspension (0.5 ml; about $1 \cdot 5 \times 10^{5}$ spores), prepared as described by Watkinson (1975), was spread over the cellophane. When incubated at $25{ }^{\circ} \mathrm{C}$ and illuminated by fluorescent strip lights $\mathrm{I} \cdot 5 \mathrm{~m}$ overhead, the mycelium made a continuous weft by 24 to $48 \mathrm{~h}$ and primordia appeared all over the surface of the plate at 5 to 7 days. In transfer experiments, 24 to 72 h-old mycelial mats, with no visible primordia, were used. Squares $(\mathrm{I} \times \mathrm{I} \mathrm{cm})$ were cut in the cellophane and each square with its attached mycelium was placed on a fresh plate of the medium to be tested.

The test media differed from the standard medium only in nitrogen or carbon source, or in the addition of a possible inhibitor. Inhibitors were sterilized by filtration, and, in quantitative experiments with glycine, serine, asparagine, glutamine, proline, hydroxyproline and glutamate, individual amino acids were autoclaved separately; these solutions were added to cool agar medium. Other media were autoclaved complete. The $\mathrm{pH}$ values of glycine, serine, glutamine and asparagine media were recorded before and after growth of the fungus.

Primordia were counted I to 5 days after transfer of samples, and samples were replicated at least four times. Only primordia developing in existing mycelium were counted; those developing round the periphery of the squares were ignored. Samples were weighed after drying overnight at $60{ }^{\circ} \mathrm{C}$.

In one experiment (Table 4 ), colonies were grown from a point inoculum on standard medium covered by cellophane. When the colony was $2 \mathrm{~cm}$ in diameter the cellophane was lifted and a well was cut in the agar beneath the edge of the mycelium. This was filled with $0.5 \mathrm{ml}$ of appropriate filter-sterilized solution, and new primordia appearing in the mycelium over the well were counted.

Developing mycelium was photographed in situ with the inverted Petri dish on the microscope stage; thus in Figs 1 and 2 the hyphae are seen from below, through the agar and Petri dish base.

\section{RESULTS AND DISCUSSION}

\section{Effects of amino acids}

In an earlier experiment (Watkinson, 1975), the number of primordia produced when undifferentiated mycelium was transferred from part of a colony to fresh medium was proportional to the logarithm of the concentration of this medium. The effect of varying the concentration of either the nitrogen or the carbon component of the medium was therefore tested. Glucose and sucrose concentrations of $80,40,20$, Io and $5 \mathrm{~g} \mathrm{l}^{-1}$ were used in the standard medium. At $48 \mathrm{~h}$ after transfer no square had more than two primordia. There was no variation in primordium number with concentration for either carbon source. However, when the casein hydrolysate concentration was varied the effect was similar to that obtained when whole medium was diluted, showing a nearly linear log dose/response relationship (Table I). Sodium nitrate, used as a nitrogen source by this fungus, did not show the same effect. To test if the effect of casein hydrolysate on the number of primordia formed was attributable to particular amino acids, the following were tested: glycine, L-serine, L-threonine, L-asparagine, L-glutamate, L-tyrosine, L-tryptophan, L-methionine, L-leucine, L-valine, L-lysine, L-glutamine, L-proline and L-hydroxyproline. Of these, tryptophan, leucine and lysine did not support growth as sole nitrogen sources; primordia were produced on glycine, serine, asparagine, glutamate, glutamine, proline and hydroxyproline. They were tested at various concentrations for a quantitative effect on primordium development. Dose/response curves were different for the seven amino acids examined in detail and only glutamate concentration had an effect similar to that of casein hydrolysate (Table I).

The $\mathrm{pH}$ values of the fresh amino-acid media containing asparagine, glycine, serine and glutamate varied between 4.7 (asparagine) and 5.9 (serine). None changed by more than $0.9 \mathrm{pH}$ units during growth of the fungus, and there was no correlation between $\mathrm{pH}$ and primordium formation or dry weight. 
Table I. Numbers of primordia developing at different concentrations of amino acids

For each medium, six squares $(\mathrm{I} \times \mathrm{I} \mathrm{cm})$ of mycelium were tested, three in each of two Petri dishes. Results for glutamine, proline and hydroxyproline are given as means with standard deviations. For casein hydrolysate, glutamate, asparagine, serine and glycine, analysis of variance with partitioning of sums of squares into orthogonal components (according to Dawkins, 1975) gave the following results when log values were used for concentrations:

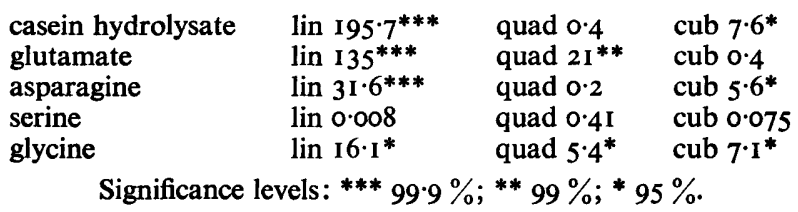

Nitrogen source
Casein hydrolysate
Monosodium
L-glutamate
L-Asparagine
L-Serine
Glycine
L-Glutamine
L-Proline
L-Hydroxyproline

No. of primordia at amino acid concn ( $\%$ nitrogen) of:

\begin{tabular}{ccccc}
\hline 0.038 & 0.075 & 0.15 & 0.3 & 0.6 \\
1.3 & 6.5 & 13.8 & 19.5 & 21.3 \\
5.3 & 4.8 & 9.3 & 21.7 & 37.0 \\
18.8 & 16.3 & 23.8 & 26.3 & 27.5 \\
10.2 & 11.3 & 10.7 & 10.5 & 10.2 \\
8.3 & 9.7 & 9.2 & 8.5 & 13.7 \\
$8.5 \pm 2.5$ & $13.7 \pm 3.5$ & $12.3 \pm 3.5$ & $10.3 \pm 1.5$ & $6.4 \pm 4.3$ \\
$21 \pm 2.3$ & $26.3 \pm 3.2$ & $19.7 \pm 1.1$ & $21.3 \pm 5.5$ & $22.3 \pm 3.6$ \\
$6.5 \pm 0.8$ & $7.6 \pm 1.6$ & $6.8 \pm 3.5$ & $12.2 \pm 4.1$ & $14 \pm 2.6$
\end{tabular}

Table 2. Dry weights of mycelium developed on agar medium containing different amino acids

After 3 days on test medium, three squares of mycelium for each treatment were separated from cellophane, pooled and dried at $60^{\circ} \mathrm{C}$ for $\mathrm{i} 8 \mathrm{~h}$.

Mycelium dry wt (mg) at amino acid concn (\% nitrogen) of:

$\begin{array}{lccccc}\text { Amino acid } & 0.038 & 0.075 & 0.15 & 0.3 & 0.6 \\ \text { Glutamate } & 29 & 30 & 35 & 36 & 38 \\ \text { Asparagine } & 23 & 25 & 37 & 37 & 43 \\ \text { Serine } & 21 & 25 & 23 & 30 & 37 \\ \text { Glycine } & 28 & 27 & 35 & 30 & 39\end{array}$

Formation of a primordium requires a supply of nutrients since it involves accumulation of reserves and growth of hyphae. However, certain amino acids appeared to have the dual function of nutrient source and stimulus to development. Tables $I$ and 2 show that these effects could occur independently; primordium numbers did not simply reflect dry weight gains. For example serine and glutamate at $0.6 \%$ nitrogen supported the same dry weight increase but glutamate gave about four times as many primordia.

The morphogenetic response to glutamate is of interest because of the regularity of the dose/response curve. Linear and quadratic components account for practically all the variation. This suggests that glutamate concentrations may be critical in initiating primordium development.

\section{Time course of coremium initiation}

Primordia were initiated at two stages. The first ones appeared at around $22 \mathrm{~h}$ after sowing the spores, when they became visible to the naked eye as white dots above the level of the mycelium. These were formed from the centres of a few chance clumps of spores, from which the germ tubes were growing out radially in all directions (Fig. I $a$ ). Soon after the appearance of these radially-oriented germ tubes, the aggregated hyphae of the coremium primordium were seen beginning to grow upwards from the centre of the 'colony'. The 


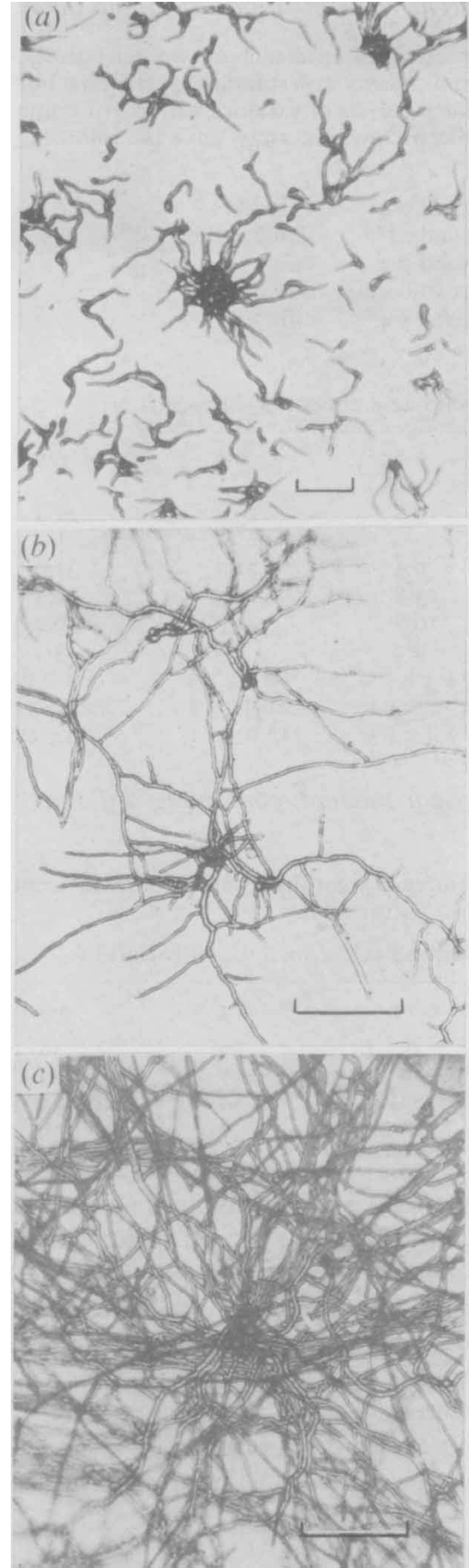

Fig. I

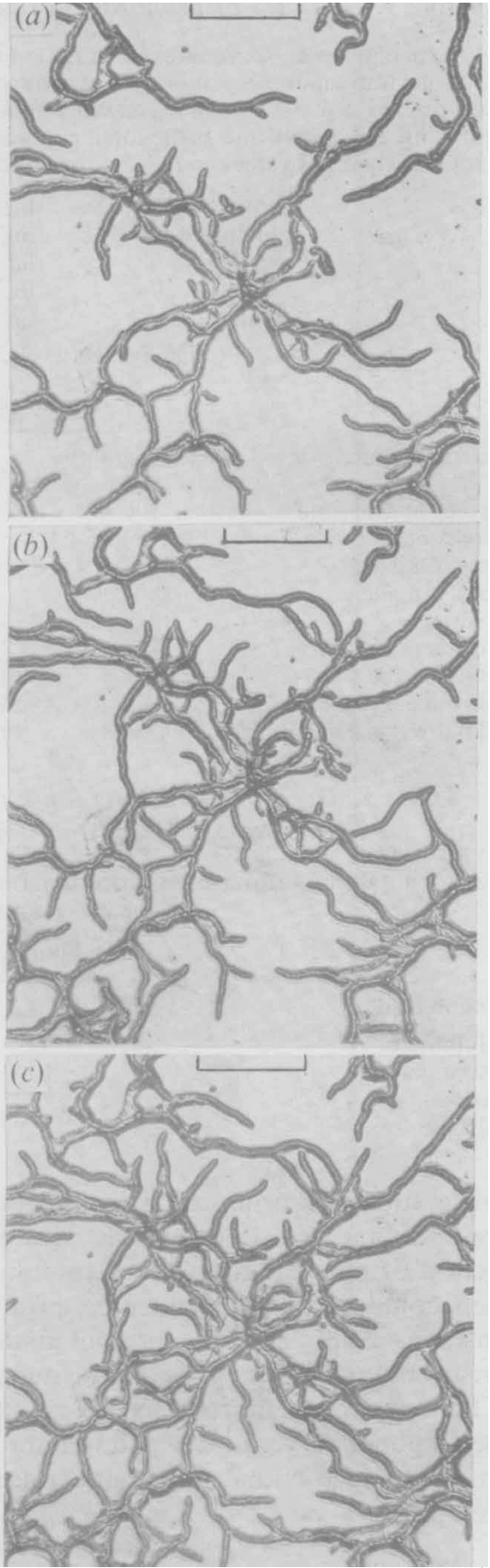

Fig. 2

Fig. I. Sites of origin of coremia: (a) from spore clumps at $24 \mathrm{~h}$ after sowing; $(b, c)$ from crossingpoints in mycelium at 20 and $35 \mathrm{~h}$ respectively. Bar markers represent $100 \mu \mathrm{m}$.

Fig. 2. Development of a coremium site in $24 \mathrm{~h}$-old mycelium: (a) at $24 \mathrm{~h} ;($ b) at $25 \mathrm{~h} ;(c)$ at $25 \mathrm{~h}$ $40 \mathrm{~min}$. Bar markers represent $100 \mu \mathrm{m}$. 
Table 3. Numbers of primordia formed after various times of exposure to inducing medium

Squares $(\mathrm{I} \times \mathrm{I} \mathrm{cm})$ were cut from evenly-seeded 24 h-old mycelium growing on cellophane over standard medium and transferred to medium containing casein hydrolysate giving a nitrogen concentration of $0.6 \%$. At intervals of up to $55.5 \mathrm{~h}$ sets of six squares were transferred to a noninducing medium containing $\mathrm{NaNO}_{3}$ (nitrogen concentration $0.6 \%$ ). Numbers of primordia in each square were counted at $55.5 \mathrm{~h}$. Control squares were placed on medium containing $\mathrm{NaNO}_{3}$ at the start of the experiments. Results are given as means for the six replicates, with standard deviations.

$\begin{array}{cc}\begin{array}{c}\text { Time on } \\ \text { inducing medium } \\ (h)\end{array} & \begin{array}{c}\text { No. of } \\ \text { primordia }\end{array} \\ 0 & \mathrm{I} \cdot 8 \pm 0 \cdot 8 \\ 2 & 0 \cdot 8 \pm \mathrm{I} \cdot 0 \\ 4.5 & \mathrm{I} \cdot 8 \pm 0 \cdot 4 \\ 7 & \mathrm{I} \cdot 5 \pm \mathrm{I} \cdot \mathrm{I} \\ 24 & 0 \cdot 2 \pm 0 \cdot 4 \\ 27 & \mathrm{I} \cdot 8 \pm \mathrm{I} \cdot 5 \\ 3 \mathrm{I} \cdot 5 & 4 \cdot 0 \pm \mathrm{I} \cdot 4 \\ 55 \cdot 5 & 7 \cdot 8 \pm 4 \cdot 8\end{array}$

Table 4. Effects of added chemicals on primordium formation in growing colonies

Colonies were grown from point inocula on cellophane over standard agar medium. When they reached $2 \mathrm{~cm}$ diam. a circular well of $\mathrm{I} \mathrm{cm}$ diam. was cut in the agar under the colony margin, filled with $0.5 \mathrm{ml}$ of test solution, and the cellophane with its overlying mycelium was replaced in contact with the solution. All new primordia visible in the mycelium over the well were counted at $24 \mathrm{~h}$ and $72 \mathrm{~h}$. All colonies continued to grow after application of the chemicals. Results are given as mean numbers of primordia for four replicates, with standard deviations.

Compound

Distilled water

Casein hydrolysate

Glucose

2-Deoxyglucose

Glucosamine

D-Gluconic acid

Cyclic AMP

Cycloheximide

Puromycin

Sodium metabisulphite
No. of primordia

$\left.\begin{array}{c}\text { Concn } \\ \text { (mg ml } \\ -1\end{array}\right)$
-
10
100
100
100
100
0.4
0.01
0.01
100

$\begin{array}{cr}\begin{array}{c}24 \mathrm{~h} \text { after } \\ \text { application }\end{array} & \begin{array}{c}72 \mathrm{~h} \text { after } \\ \text { application }\end{array} \\ 2 \cdot 0 \pm 0 \cdot 9 & 2 \cdot 5 \pm \mathrm{I} \cdot 3 \\ \mathrm{I} \cdot 3 \pm \mathrm{I} \cdot 5 & 2 \cdot 5 \pm \mathrm{I} \cdot 0 \\ \mathrm{I} \cdot 5 \pm 2 \cdot 4 & 3 \cdot 5 \pm \mathrm{I} \cdot 3 \\ 0 & 0 \\ 0 & 15 \cdot 0 \pm 2 \cdot 2 \\ 0 & 6 \cdot 5 \pm 4 \cdot 5 \\ 4.5 \pm 2 \cdot 8 & 6 \cdot 0 \pm 0.8 \\ 0 & 4 \cdot 0 \pm \mathrm{I} \cdot 8 \\ 0.75 \pm 0.9 & 4 \cdot 0 \pm 0.8 \\ 0 & 1 \cdot 8 \pm \mathrm{I} \cdot 7\end{array}$

majority of primordia, however, did not develop from clumps of spores, but appeared after the mycelium had covered the plate. A prerequisite for formation of a coremium was the establishment of a colony-like pattern of hyphae, with a centre subtended by radiating hyphae. Figure $2(a, b, c)$ shows such a centre developing in $24 \mathrm{~h}$-old mycelium over a period of $100 \mathrm{~min}$. As the germlings fused, a reticulum of main hyphae developed and centres appeared sometimes at the site of germination of a group of spores and sometimes at crossing-points in the network (Fig. I $b, c$ ). After $48 \mathrm{~h}$ the mycelium became too dense for further observations. The coremia initiated from a reticulate mycelium emerged fairly synchronously and regularly spaced, at times depending on the composition of the medium. No further coremia appeared after the main flush. On standard medium, primordia appeared at 5 to 7 days but at high amino-acid concentrations they appeared only $24 \mathrm{~h}$ after transfer of $24 \mathrm{~h}$-old mycelium to the appropriate medium.

Table 3 shows the time taken for amino acids to influence coremium initiation. About $30 \mathrm{~h}$ contact with medium rich in amino acid was needed for an effect, and a much greater effect was obtained at $55 \mathrm{~h}$ exposure. During the exposure period, the mycelium was still 
Table 5. Effects of added chemicals on primordium initiation in 24 h-old mycelium

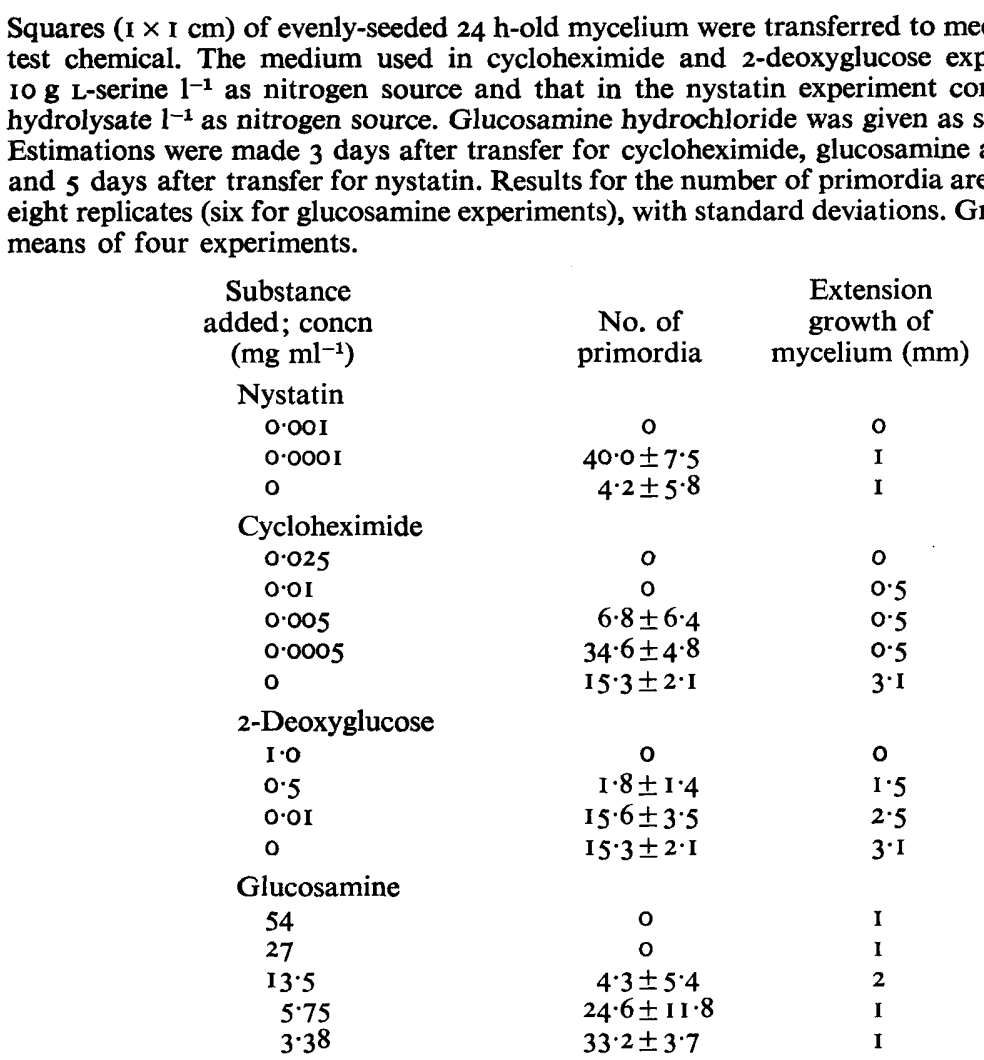

undergoing fusion between germlings and new centres were developing. Numerous possible sites for coremium initiation were, however, already discernible in the 24 h-old mycelium. Since the availability of amino acids after this stage could still determine how many primordia were formed in the mycelium, it is likely that amino acids act by promoting primordium development at these potential sites rather than by causing the sites themselves to be formed. The proportion that develop into coremia depends on the availability of the appropriate stimulus.

Mycelium returned to non-inducing nutrient medium after brief exposure to inducing medium was not stimulated (Table 3), and so the stimulus was not an irreversible 'trigger'. The exposure times and concentrations required for an effect also seem to rule"out mechanisms involving cell surface receptors or effects on uptake rates. It is more probable that morphogenesis is affected by changes in level of internal free amino acids or related metabolites.

\section{Effects of added chemicals}

The effect of some possible inhibitors on primordium development is shown in Tables 4 and 5. In one experiment (Table 4), test solutions were administered to part of a colony as it grew over a nutrient medium (see Methods). Glucosamine hydrochloride, D-gluconic acid and cyclic AMP increased the numbers of primordia formed, while 2-deoxyglucose completely inhibited primordium development. Table 5 shows the effects on transferred squares of synchronously-growing young mycelium of four inhibitors incorporated in nutrient medium. Nystatin, cycloheximide and glucosamine hydrochloride, at sublethal concentrations, accelerated and also increased primordium formation. When squares of 
mycelium were transferred back to fresh nutrient medium from a medium containing cycloheximide at a concentration that inhibited both growth and differentiation $(0.025 \mathrm{mg}$ $\mathrm{ml}^{-1}$ ), there was a sudden production of large numbers of small primordia ( $2 \mathrm{I} \pm \mathrm{15} \cdot 3$ $\mathrm{cm}^{-2}$ ). This suggests that some process leading to primordium formation was able to continue in the presence of cycloheximide, even though growth was prevented. Possibly this process is amino-acid uptake. Hunter \& Segel (197I) found that cycloheximide treatment of Penicillium chrysogenum increased internal free lysine and ascribed this to its different effects on uptake and utilization of the amino acid.

Primordium formation was prevented by 2-deoxyglucose at a concentration that allowed extension of undifferentiated hyphae over the surface of the medium. This might be due to interference by 2-deoxyglucose with the carbohydrate biosynthesis accompanying primordium formation. Glucosamine hydrochloride was used by the fungus as sole nitrogen source and appeared to inhibit primordium development at high concentrations and to promote it at low concentrations. Nystatin can interfere with amino-acid transport (Stachiewicz \& Quastel, 1963); here, damage to some cell membranes might have stimulated primordium formation by increasing amino-acid availability to others.

The regulation of primordium development in colonies in nature presumably cannot involve very high external amino-acid concentrations. However, the fact that primordia can be stimulated to develop by this means suggests that their development is controlled via amino-acid metabolism. Development might be initiated above a critical intrahyphal level of certain amino acids or their metabolites and this would accord with Wright's (1973) view that differentiation may be controlled by means of critical substrate levels.

In a colony growing from a point inoculum coremia often develop in a pattern of concentric rings. The mycelium between these rings develops coremia if transferred to nutrient medium (Watkinson, 1975). This implies that potential sites for primordium formation receive positional information (as defined by Wolpert, 197I) concerning their relation to the colony margin and to older coremia in the mycelium. The present results suggest that a concentration gradient of exogenous or endogenous free amino acid could fulfil this role. Such a gradient could be maintained by differences in rates of uptake, utilization and leakage by mycelium of different ages. The periodicity of initiation could result from the need for a threshold concentration of amino acids for primordium formation.

The able technical assistance of Miss L. Bromley is gratefully acknowledged.

\section{REFERENCES}

Carlile, M. J., Lewis, B. G., Mordue, E. M. \& Northover, J. (I96I). The development of coremia. I. Penicillium claviforme. Transactions of the British Mycological Society 44, 129-133.

DawkIns, H. C. (1975). Statforms. London: Edward Arnold.

Faraj Salman, A.-G. (1970). Einfluss von Licht auf die Koremienbildung und ihre kreisförmige Anordnung. Kulturpflanze 18, 89-97.

FaraJ Salman, A.-G. (I97I $a$ ). Zur Induktion einer endogenen Rhythmik bei Mutanten des Pilzes Penicillium claviforme Bainier. I. Wirkungsweise von Alkoholen. Archiv fur Protistenkunde rr3, 306-313.

Faraj Salman, A.-G. (I97 l b). Zur Induktion einer endogenen Rhythmik bei Mutanten des Pilzes Penicillium claviforme Bainier. II. Wirkungsweise von Detergenten. Biochemie und Physiologie der Pflanzen 162, 470-473.
Hunter, D. R. \& Segel, I. H. (I97I). Acidic and basic amino acid transport systems of Penicillium chrysogenum. Archives of Biochemistry and Biophysics x44, 168-183.

STACHIEWICZ, E. \& QUASTel, J. H. (1963). Amino acid transport in yeasts and the effects of nystatin. Canadian Journal of Biochemistry and Physiology 4x, 397-407.

WATKINSON, S. C. (1975). Regulation of coremium morphogenesis in Penicillium claviforme. Journal of General Microbiology 87, 292-300.

WOLPERT, L. (I97I). Positional information and pattern formation. Current Topics in Developmental Biology 6, I83-224.

Wright, B. E. (1973). Critical Variables in Differentiation. Englewood Cliffs, New Jersey: PrenticeHall. 